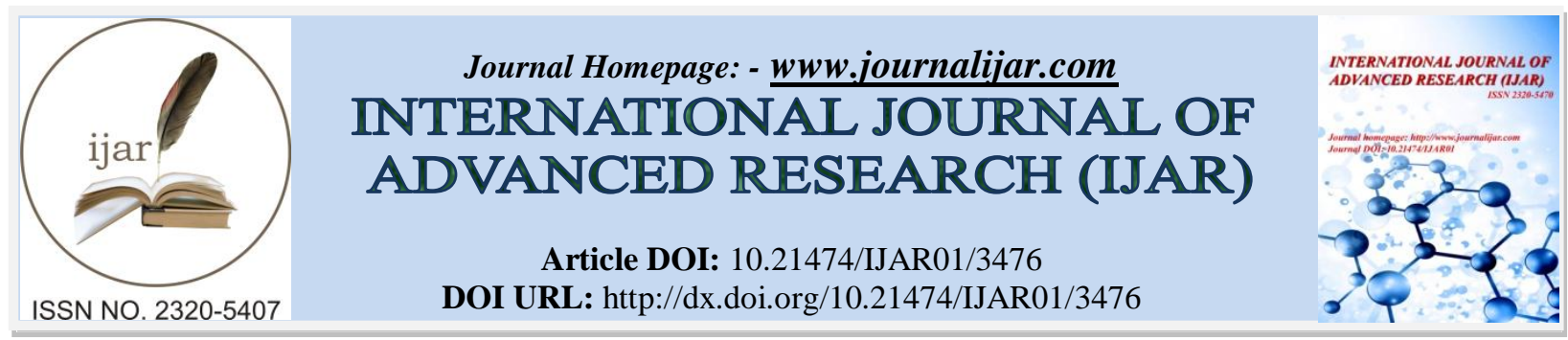

RESEARCH ARTICLE

\title{
FORMULATION DEVELOPMENT OF MUCOADHESIVE BILAYER TABLET WITH METFORMIN HYDROCHLORIDE.
}

Sabnin Shair, Nafisa Nowal Rafa, Md. Abu Sufian, A. H. M. Nazmul Hasan and "Md. Asaduzzaman.

Department of Pharmacy, University of Asia Pacific, Dhaka-1215, Bangladesh.

\section{Manuscript Info}

Manuscript History

Received: 02 January 2017

Final Accepted: 05 February 2017

Published: March 2017

Key words:-

Mucoadhesion, gastroretentive, sustained release, kinetic model, exvivo study.

\section{Abstract}

In the present study, an attempt was made to design and evaluate bilayer gastroretentive drug delivery system exhibiting a combination of an immediate release layer and a sustained release mucoadhesive layer as second layer to prolong residence time in stomach using Metformin $\mathrm{HCl}$ as a model drug to reduce frequency of administration and to overcome bioavailability problems. A total of 5 formulations of bilayer tablets containing the drug were prepared by direct compression method using 5 different mucoadhesive polymers in 5 different formulations $\left(\mathrm{F}_{1}-\mathrm{F}_{5}\right)$ respectively to impart mucoadhesion. Formulated bilayer tablets were evaluated by different parameters such as; ex vivo mucoadhesion strength, ex vivo mucoadhesion time, In vitro drug release study. In vitro drug release data were fitted to various kinetic models (Zero order, $1^{\text {st }}$ order, Higuchi model, Korsmeyer- Peppas model). Upon $\mathrm{R}^{2}$ value analysis it was observed that release from $\mathrm{F}_{1}$, $\mathrm{F}_{3}, \mathrm{~F}_{5}$ followed Higuchi model and $\mathrm{F}_{2}, \mathrm{~F}_{5}$ followed Korsmeyer- Peppas kinetic model. Mechanism of drug release was found to be Fickian diffusion (value of $\mathrm{n}<0.45$ ). $\mathrm{F}_{4}$ with $\mathrm{Na} \mathrm{CMC}$ was found to be more promising in ex vivo mucoadhesion study showing residence time of $4.08 \mathrm{hrs}$. This study concludes that formulated bilayer tablets were satisfactory in providing prompt release from $1^{\text {st }}$ layer then maintained sustained release effect with promising mucoadhesion behavior in stomach.

Copy Right, IJAR, 2017,. All rights reserved.

\section{Introduction:-}

The new drug delivery system with better efficacy and safety with reduced dosing frequency and improved patient compliance is the current area of research by formulation development scientists. Tablet being most preferred dosage form for its ease of manufacturing and patient convenience is always a first choice of dosage form. The single layer tablets leads to frequent dosing and unpredicted plasma level for drugs with shorter half-lives. Number of diseases require immediate release of drug for instant effect and then plasma concentration has to be maintained for prolong effect of the drug. So, in order to deliver drugs in a predictable time frame, multilayered oral controlled release delivery systems are designed. These systems enhance the efficacy, minimize the adverse effects and increase the bioavailability of drugs. 
Mucoadhesive drug delivery is a topic of interest in the design of drug delivery systems to prolong the residence time of the dosage form at the site of application or absorption and thereby to facilitate the intimate contact of dosage form, thus to improve and enhance the bioavailability.

Dosage forms designed for mucoadhesive drug delivery should be small and flexible enough to be acceptable for patients and should not cause irritation. Other desired characteristics of a mucoadhesive dosage form include high drug loading capacity, controlled drug release (preferably unidirectional release), good mucoadhesive properties, smooth surface, tastelessness, and convenient application. Erodible formulations can be beneficial because they do not require system retrieval at the end of desired dosing interval. A number of relevant mucoadhesive dosage forms have been developed for a variety of drugs (Boddupalli et al., 2010).

The mechanism of adhesion of certain macromolecules to the epithelium of a mucous tissue is understood. They are characterized with an epithelial level whose surface is protected by mucus. The mucus contains glycoproteins, lipids, inorganic salts and $95 \%$ water by mass, making it a highly hydrated system. Mucin is the significant glycoprotein of mucus and is responsible for its structure. The principal functions of mucus are covering and lubricating the epithelium and some other functions based on the epithelium protection. Mucus width can change from 50-450 $\mu \mathrm{m}$ in the stomach to less than $1 \mu \mathrm{m}$ in the oral cavity (Smart, 2005).

The mucous area, majorly used for the drug administration and absorption, is gastrointestinal mucus. The mucoadhesion ought to extend over the substrate to initiate the close connection, enhance the surface contact, and in turn increase the diffusion of its chains inside the mucus. The mechanism of mucoadhesion is usually separated in two stages, the contact stage and the consolidation stage. The first stage is characterized by the contact between the mucoadhesion and the mucous membrane, with covering and swelling of the formulation and initiating its deep contact with the mucous level (Hägerstrom and Edsman, 2003).

The development of sustain release dosage form can achieve the aim of releasing the drug slowly for a long period but this is not sufficient to get sustained therapeutic effect. They may be cleared from the site of absorption before emptying the drug content. Instead, the mucoadhesive dosage form will serve both the purposes of sustain release and presence of dosage form at the site of absorption.

In the recent times, multi-layer matrix tablets are gaining importance in the design of oral controlled drug delivery systems. Bi-layer tablets are novel drug delivery systems where combinations of two or more drugs are given in a single unit. They are preferred for the following reasons: To co-administer two different drugs in the same dosage form, to minimize physical and chemical incompatibilities, for staged drug release, IR and SR in the same tablet, for chronic condition requiring repeated dosing.

In the present research, an attempt was made to develop oral mucoadhesive bilayered tablet of Metformin $\mathrm{HCl}(\mathrm{MH})$ using Xanthan gum, NaCMC, HPMC 100CPS, HPMC (K-100LV) and Methocel K4M polymers. Metformin HCl, chemically N,N-Dimethyl imido dicarbonimidic diamide, is a biguanide anti hyperglycemic agent used for treating non-insulin-dependent diabetes mellitus (NIDDM). It improves glycemic control by decreasing hepatic glucose production, decreasing glucose absorption and increasing insulin-mediated glucose uptake. Metformin is the only oral anti hyperglycemic agent that is not associated with weight gain. Metformin may induce weight loss and is the drug of choice for obese NIDDM patients. When used alone, metformin does not cause hypoglycemia; however, it may potentiate the hypoglycemic effects of sulfonylurea's and insulin. The half-life of metformin is $6.2 \mathrm{hrs}$ (Kadhe and Arasan, 2002; Desvergne and Wahli, 1999; Berger and Moller, 2002). It is freely soluble in water, slightly soluble in alcohol, practically insoluble in acetone and in methylene chloride (Chawla et al., 200; Chinetti et al., 2000; Willson et al., 2001).

It is a hydrophilic drug and is slowly and incompletely absorbed from the gastrointestinal tract, and the absolute bioavailability of a single $500 \mathrm{mg}$ dose is reported to be $50 \%$ to $60 \%$. This indicates the need to develop dosage forms that can retain the drug in the stomach for better absorption. An obstacle to more successful use of metformin therapy is the high incidence of concomitant gastrointestinal symptoms, such as abdominal discomfort, nausea, and diarrhea that especially occur during the initial weeks of treatment. The compound also has relatively short plasma elimination half- life (1.5 - 4.5hrs). Sustained release formulations may be administered once or twice daily. By considering above parameters, mucoadhesive bilayer tablet of metformin may be utilized. 
This study describes the preparation of bilayer device comprising Metformin $\mathrm{HCl}$ as API and mucoadhesive layer of polymer to prolong its duration of action and improve patient compliance.

\section{Materials and methods:-}

\section{Materials:-}

Metformin $\mathrm{HCl}$ was received as a gift sample from Square Pharmaceutical Ltd, Bangladesh.; Methocel was purchased from Colorcon; Hydrochloric acid was obtained from Merck, Germany; and Xanthan gum, NaCMC, HPMC 100CPS, HPMC (K-100LV), Lactose, Magnessium stearate, Talc and Aerosil were obtained from local market. All other ingredients were of analytical grade.

Methods:-

Preparation of bilayer tablet:-

A total of 5 different types of formulations were prepared in 2 stages using a $13 \mathrm{~mm}$ - diameter die on an infrared hydraulic press with $5 \mathrm{~N}$ compression force for a total of 1 minute 30 seconds. Initially the mucoadhesive layer was prepared according to Formulation plan (Table-1) for 30 seconds by simple thumb pressure then the $2^{\text {nd }}$ layer (immediate release layer) was prepared by final compression force of $5 \mathrm{~N}$ for 1 minute.

Table 1:- Formulation plan:

\begin{tabular}{|c|c|c|c|c|c|c|}
\hline \multirow{2}{*}{$\begin{array}{l}\text { Ingredients } \quad(\mathrm{mg} / \\
\text { tablet })\end{array}$} & \multirow{2}{*}{$\begin{array}{l}\text { Immediate release layer } \\
\left(F_{1}-F_{5}\right) \text { Composition is same } \\
\text { for all formulations }\end{array}$} & \multicolumn{5}{|c|}{ Mucoadhesive sustained release layer } \\
\hline & & $\mathbf{F}_{1}$ & $\mathbf{F}_{2}$ & $\mathbf{F}_{3}$ & $\mathbf{F}_{4}$ & $\mathbf{F}_{5}$ \\
\hline Metformin $\mathrm{HCl}$ & 150 & 250 & 250 & 250 & 250 & 250 \\
\hline HPMC100cps & - & 200 & - & - & - & - \\
\hline HPMC(K-100LV) & - & - & 200 & - & - & - \\
\hline $\mathrm{NaCMC}$ & - & - & - & 200 & - & - \\
\hline Methocel K4M & - & - & - & - & 200 & - \\
\hline Xanthan gum & - & - & - & - & - & 200 \\
\hline Lactose & 100 & 50 & 50 & 50 & 50 & 50 \\
\hline Magnesium stearate & 1 & 1 & 1 & 1 & 1 & 1 \\
\hline Talc & 1 & 1 & 1 & 1 & 1 & 1 \\
\hline Aerosil & 1 & 1 & 1 & 1 & 1 & 1 \\
\hline Total weight & 253 & 503 & 503 & 503 & 503 & 503 \\
\hline
\end{tabular}

Evaluation of physical and biological parameters of formulated bilayer tablets:-

In vitro drug release study for mucoadhesive bilayer tablet:-

In vitro release profile for each formulated bilayer tablet was performed using USP type II dissolution apparatus (Electrolab, Mumbai, India). Dissolution studies were carried at rotation speed of $50 \mathrm{RPM}$, in $900 \mathrm{ml}$ of $0.01 \mathrm{~N} \mathrm{HCl}$ of pH 2.0 at $37 \pm 0.5^{\circ} \mathrm{C}$. Aliquot of $5 \mathrm{ml}$ was withdrawn upto $8 \mathrm{hrs}$. The withdrawn volume was replaced with same volume of dissolution medium in order to keep the total volume constant. The absorbance of sample was measured by an UV spectrophotometer at $233 \mathrm{~nm}$ after suitable dilution if necessary. After filtration, the amount of drug release was determined from the standard calibration curve of pure drug (Singh et al., 2012).

Kinetic modeling of in vitro drug dissolution profile:-

In vitro drug release data of all the bilayer tablet formulations of Metformin $\mathrm{HCl}$ was subjected to goodness of fit test by linear regression analysis according to zero order, first order kinetics and according to Higuchi's and Korsmeyer-Peppas models to ascertain mechanism of drug release.

\section{Measurement of ex-vivo mucoadhesive strength:-}

Bioadhesive strength of the tablets was measured on modified physical balance. A piece of goat stomach mucosa was pasted to a petri-dish with cyanoacrylate adhesive and the mucus membrane was wetted with 2-3 drops of 0.01 $\mathrm{N} \mathrm{HCl}$ media. The tablets were tied with thread and attached with the mucous membrane. Another end of thread tied with one side of the physical balance. The weight required to detach the tablet from the mucosal surface was taken as the measure of mucoadhesive strength. Force of adhesion was calculated from the mucoadhesive strength as per following equation: 
Force of adhesion $(\mathrm{N})=($ mucoadhesive strength $\times 9.81) \div 1000$ :

Measurement of ex-vivo residence time:-

The ex-vivo residence time was determined using a locally modified USP disintegration apparatus. A segment of goat stomach mucosa was glued to the surface of the beaker, vertically attached to the apparatus. The mucoadhesive tablet was then pasted to the mucosal membrane by applying a light force with a finger trip for 30 seconds. Then the

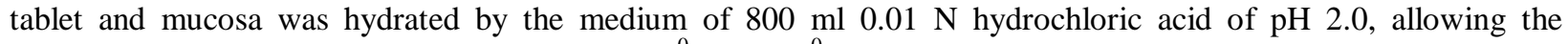
apparatus to move up and down maintaining $37^{0} \mathrm{C} \pm 0.5^{0} \mathrm{C}$; monitoring the tablet adhesion for 8 hours to see retention time and correlate with sustaining property of formulation. The time for the tablet to detach from the goat stomach mucosa was recorded as the mucoadhesion time (Umarji et al., 2012).

\section{Results and Discussion:-}

The main goal of this work was to develop new mucoadhesive bilayer tablets of metformin $\mathrm{HCl}$; consisting of an immediate release and a of sustained release layer. The double layered structure design was expected to provide immediate release of metformin $\mathrm{HCl}$ from the outer layer to produce a prompt pharmacological action then sustained release from second layer to provide a sustained release of enough drug over an extended period of time and retain in stomach for an extended period of time.

A total of 5 formulations of mucoadhesive bilayer tablets were prepared and evaluated for biological and physical parameters. The composition of final bilayer formulation had been chosen from the previous single sustained release layer formulation which gives the best average percent release and best fitted with kinetics model of dissolution release pattern. Different amounts $(150 \mathrm{mg}, 180 \mathrm{mg}, 200 \mathrm{mg}, 220 \mathrm{mg}$ ) of 5 different mucoadhesive polymers (HPMC 100 cps, HPMC K-100LV, Xanthun gum, NaCMC, Methocel K4M) were used to prepare single sustained release layer. The study indicated that increase of concentration of polymer decreases In vitro drug release and gives larger $\mathrm{T}_{50 \%}$ value; which indicates more sustained release effect. Thus the middle amount of polymers (200 $\left.\mathrm{mg}\right)$ was selected for the final bilayer formulation.

According to work plan, the formulated bilayer tablets were evaluated for their in vitro drug release profile study, $e x$ vivo mucoadhesive strength, ex vivo residence time, $\mathrm{T}_{50 \%}, \mathrm{~T}_{25 \%}, \mathrm{~T}_{70 \%}$ determination.

\section{Physical appearance:-}

The appearance of bilayer tabets was smooth and uniform on physical examination.

Kinetic modeling of in vitro drug dissolution profile:-

In vitro drug release studies were conducted and fitted to different kinetic models. Cumulative percent of drug release from the formulations upto $8 \mathrm{hrs}$ is shown in the Figure-1; release rate kinetic constants and $\mathrm{R}^{2}$ values obtained from different kinetic models are tabulated in the following Table 2.

Table 2:- Interpretation of release rate constants and $\mathrm{R}^{2}$ values for different release kinetics of formulated bilayer tablets:

\begin{tabular}{|l|l|l|l|l|l|l|l|l|}
\hline \multirow{2}{*}{ Formulations } & \multicolumn{2}{l|}{ Zero order } & \multicolumn{2}{l|}{ First order } & \multicolumn{2}{l|}{ Higuchi } & \multicolumn{2}{l|}{ Korsmeyer } \\
\cline { 2 - 9 } & $\mathbf{K}$ & $\mathbf{R}^{2}$ & $\mathbf{K}_{1}$ & $\mathbf{R}^{2}$ & $\mathbf{K}_{\mathbf{H}}$ & $\mathbf{R}^{2}$ & $\mathbf{N}$ & $\mathbf{R}^{2}$ \\
\hline $\mathrm{F}_{1}$ & 5.935 & 0.873 & -0.040 & 0.940 & 19.25 & 0.978 & 0.426 & 0.956 \\
\hline $\mathrm{F}_{2}$ & 7.084 & 0.839 & -0.059 & 0.950 & 23.36 & 0.971 & 0.328 & 0.988 \\
\hline $\mathrm{F}_{3}$ & 6.614 & 0.864 & -0.050 & 0.940 & 21.48 & 0.970 & 0.370 & 0.962 \\
\hline $\mathrm{F}_{4}$ & 6.648 & 0.883 & -0.048 & 0.953 & 21.46 & 0.980 & 0.414 & 0.964 \\
\hline $\mathrm{F}_{5}$ & 6.851 & 0.854 & -0.054 & 0.948 & 22.42 & 0.974 & 0.354 & 0.978 \\
\hline
\end{tabular}




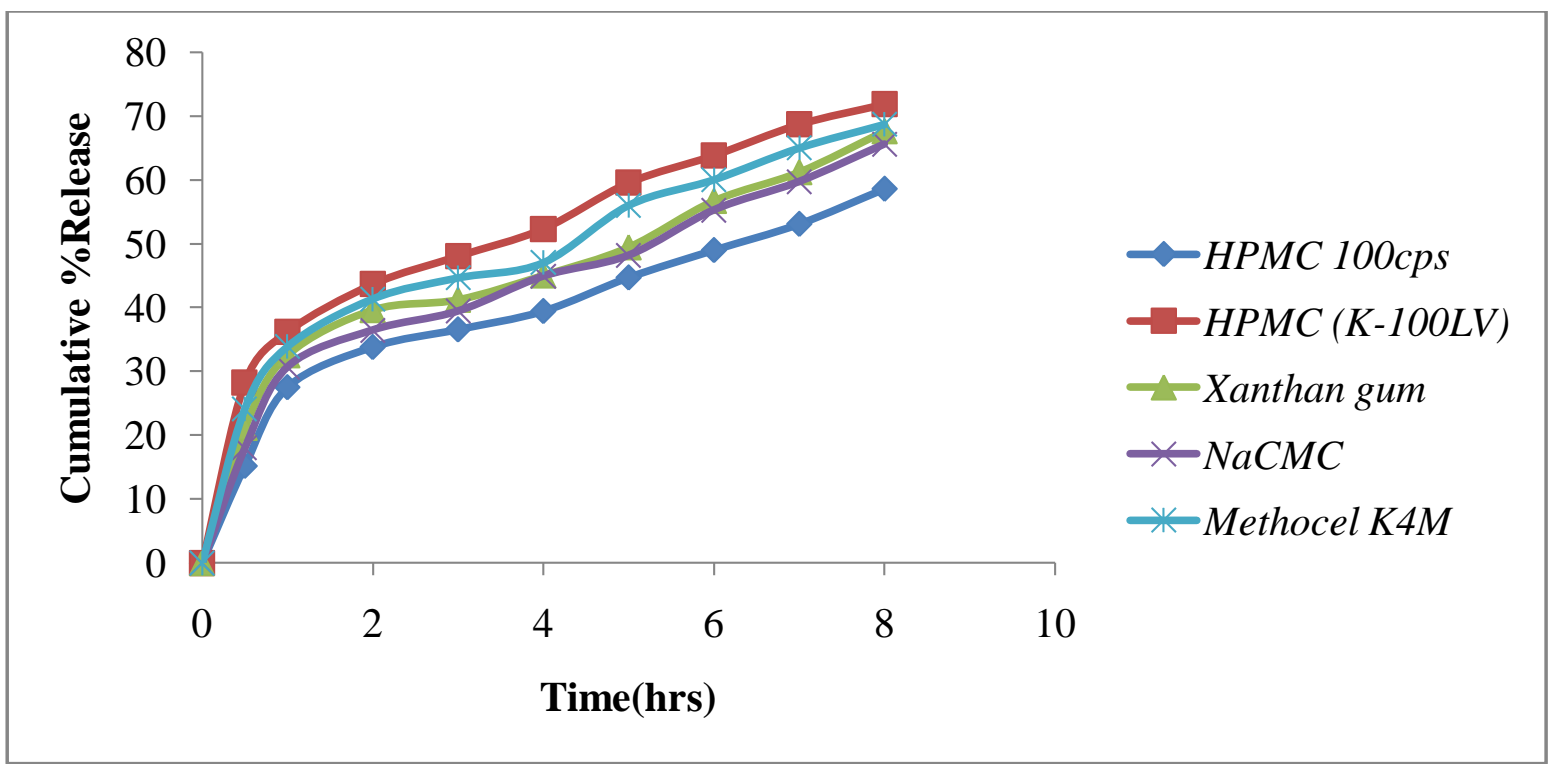

Fig 1:- In vitro release profile of formulated bilayer tablets containing different types of polymers. Here, used mucoadhesive polymer indicated the formulations. $F_{1}$ is formulated with HPMC $100 \mathrm{cps}, \mathrm{F}_{2}$ is formulated with HPMC (K-100LV), $F_{3}$ is formulated with Xanthun gum, $F_{4}$ is formulated with NaCMC, $F_{5}$ is formulated with Methocel K4M.

The in vitro release data are treated with different model of release kinetics in the above Table 2 and the best fitted model was explained by the help of $\mathrm{R}^{2}$ value in Table 3 given below. Evaluated, $\mathrm{T}_{25 \%}, \mathrm{~T}_{50 \%}, \mathrm{~T}_{70 \%}$ were also tabulated in the Table 3 and $n$ values were also mentioned for drug transport mechanism explanation. Fig 2 illustrates the evaluated values of successive fractional dissolution time of formulated bilayer tablets.

Table 3:- Drug release kinetics and dissolution parameters of formulated bilayer tablets:

\begin{tabular}{|l|l|l|l|l|l|l|l|}
\hline Formulations & Best Fit Model & $\mathbf{R}^{\mathbf{2}}$ & $\mathbf{n}$ & $\mathbf{K}_{\mathbf{H}}$ & $\mathbf{T}_{\mathbf{2 5}}$ & $\mathbf{T}_{\mathbf{5 0} \%}$ & $\mathbf{T}_{\mathbf{7 0} \%}$ \\
\hline $\mathrm{F}_{1}$ & Higuchi & 0.978 & 0.426 & 19.25 & 1.18 & 5.98 & 18.03 \\
\hline $\mathrm{F}_{2}$ & Korsmeyer & 0.988 & 0.328 & - & 0.35 & 2.93 & 12.27 \\
\hline $\mathrm{F}_{3}$ & Higuchi & 0.970 & 0.370 & 21.48 & 0.66 & 4.32 & 15.37 \\
\hline $\mathrm{F}_{4}$ & Korsmeyer & 0.978 & 0.414 & - & 0.88 & 4.69 & 14.6 \\
\hline $\mathrm{F}_{5}$ & Higuchi & 0.980 & 0.354 & 22.42 & 0.51 & 3.62 & 13.67 \\
\hline
\end{tabular}

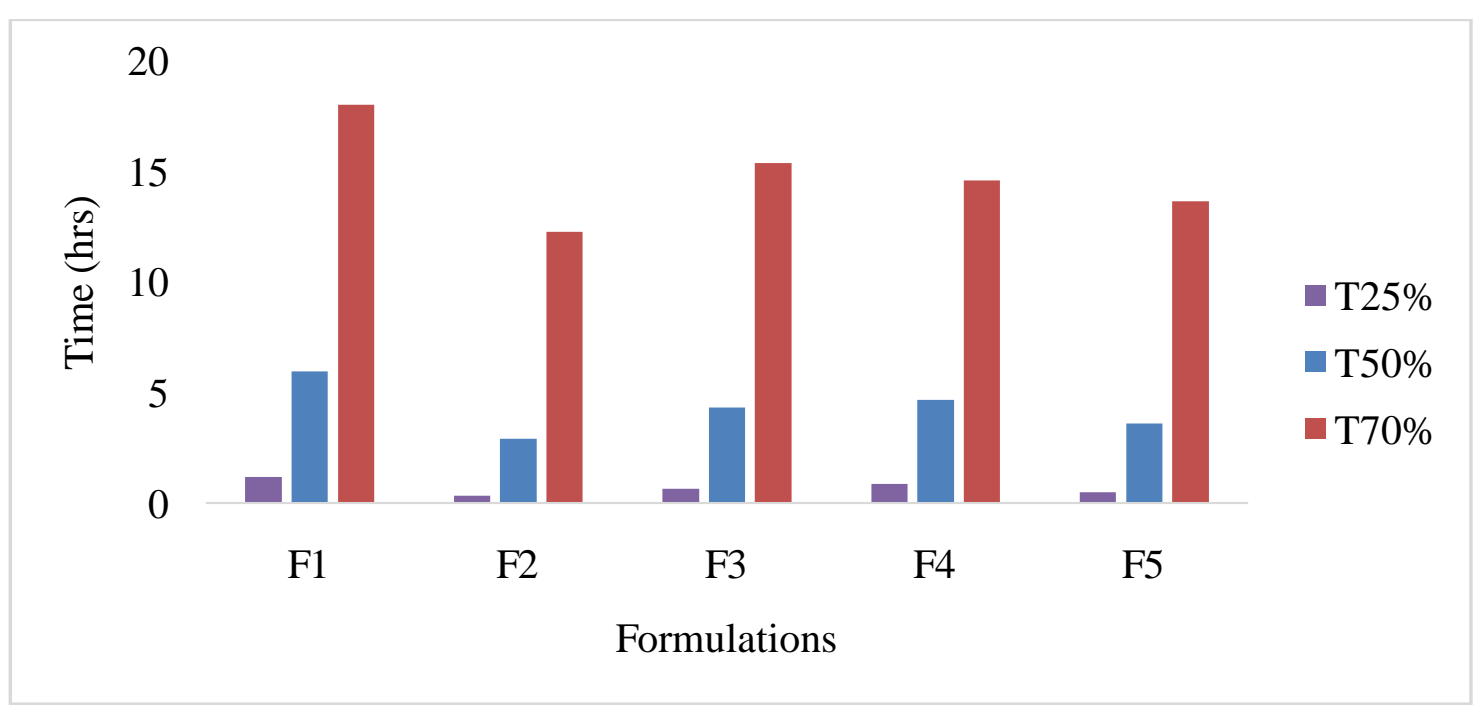

Fig 2:- Successive fractional dissolution time of formulated bilayer tablets. 
$\mathrm{T}_{50 \%}$ values of formulations manifest the drug release rate of dosage form and the retarding efficacy of polymers. Higher $\mathrm{T}_{50 \%}$ value indicates a higher drug retarding ability of polymer and vice versa. Fig - 2 indicates better sustained release effect was found in $\mathrm{F}_{1}$ and $\mathrm{F}_{3}$ compared to other formulations.

Diffusional release mechanism of the formulations was interpreted from in vitro drug release data by analyzing the $\mathrm{n}$ values by fitting in Korsmeyer Peppas kinetic model and results are tabulated in Table 4.

Table 4:- Interpretation of diffusional release mechanism from in vitro drug release data from formulated bilayer tablets:

\begin{tabular}{|c|c|c|}
\hline Formulation & n (Release exponent) & Drug Transport Mechanism \\
\hline $\mathrm{F}_{1}$ & 0.426 & Fickian diffusion \\
\hline $\mathrm{F}_{2}$ & 0.328 & Fickian diffusion \\
\hline $\mathrm{F}_{3}$ & 0.370 & Fickian diffusion \\
\hline $\mathrm{F}_{4}$ & 0.414 & Fickian diffusion \\
\hline $\mathrm{F}_{5}$ & 0.354 & Fickian diffusion \\
\hline
\end{tabular}

Ex-vivo study for evaluation of mucoadhesive behavior of bilayer tablets:-

The Ex-vivo mucoadhesive strength of the tablets was determined for different formulations using goat stomach mucosa. Ex vivo residence time was evaluated by conducting a study for $8 \mathrm{hrs}$ by recording the time for complete detachment of tablet from mucosal surface. The evaluation results are tabulated in Table 5 and illustrated in Fig 3 , Fig 4.

Table 5:- Ex- vivo evaluation of mucoadhesive behavior of formulated bilayer tablets:

\begin{tabular}{|l|l|l|}
\hline Formulations & Detachment force $(\mathbf{N})$ & Residence time (hrs) \\
\hline$F_{1}$ & 0.661 & 2.22 \\
\hline$F_{2}$ & 0.621 & 1.4 \\
\hline$F_{3}$ & 0.6 & 3.07 \\
\hline$F_{4}$ & 0.916 & 4.08 \\
\hline$F_{5}$ & 0.523 & 2.02 \\
\hline
\end{tabular}

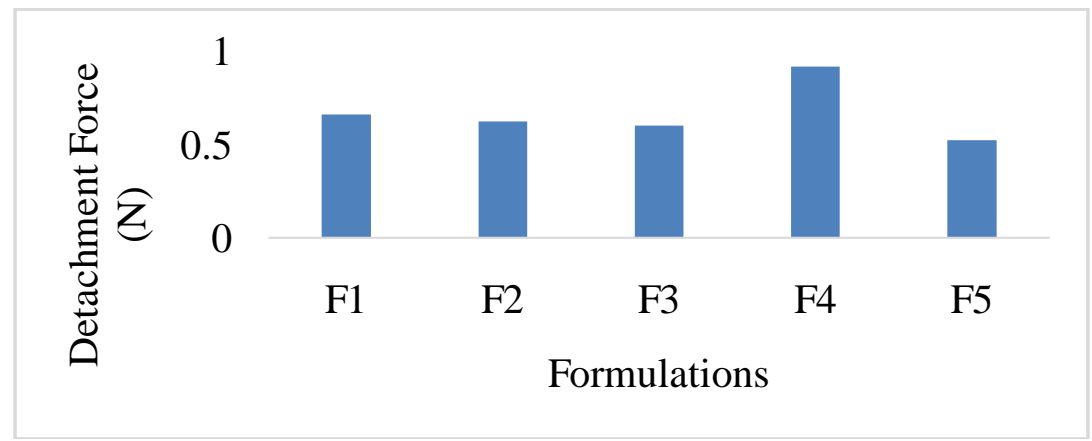

Fig 3:- Detachment force of different formulation containing different mucoadhesive polymer.

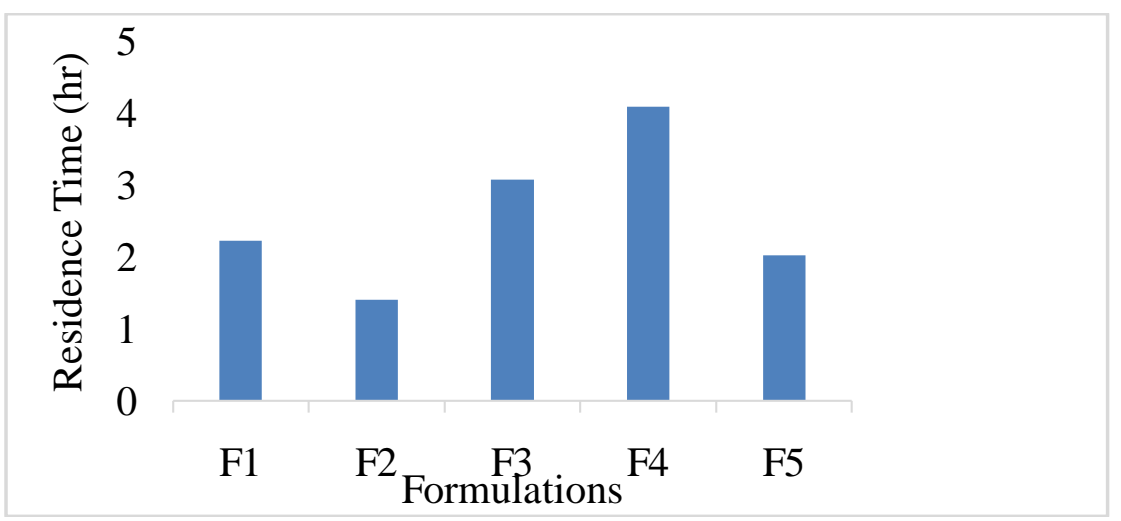

Fig 4:- Ex vivo residence time of different formulation containing different ratio of mucoadhesive polymer. 
From the Table 4, Fig 3 and Fig 4; it was observed that the highest mucoadhesive strength was observed with the formulation showing highest detachment force. $\mathrm{F}_{4}$ containing $\mathrm{NaCMC}$ showed highest mucoadhesive strength. Tablet containing an increased concentration of polymer showed higher mucoadhesion with long residence time (4.08 hrs). This finding is owing to the hydrophillic nature of $\mathrm{NaCMC}$; it is hydrated easily with less contact time and forms a strong interpenetrating network with mucin molecules because of the polymer's long linear chain structure. A linear increase in mucoadhesion was observed with an increase in contact time.

\section{Conclusion:-}

In the present study, 5 different formulations of mucoadhesive bilayer tablet of metformin $\mathrm{HCl}\left(\mathrm{F}_{1}-\mathrm{F}_{5}\right)$ were prepared by using 5 different Polymers by direct compression method. The results of in-vitro drug release profile of bilayered tablets were satisfactory. But, $\mathrm{F}_{1}$ and $\mathrm{F}_{4}$ provided better controlled and sustained In vitro drug release compared to other three formulations. The $\mathrm{T}_{50 \%}$ values of these five formulations which is an important parameter for knowing the retarding ability of drugs were also found satisfactory. Results from ex vivo evaluation of mucoadhesive behavior of these five formulations were also good which provides increased residence time of drug in GIT and gave clear understanding about the bioadhesion properties of the bilayer tablet. However, an appropriate balance between various levels of the five polymers is needed to acquire proper release and mucoadhesion. Finally, it can be concluded that by formulating mucoadhesive bilayer tablets of metformin $\mathrm{HCl}$, its complete release can be ensured prior to absorption and hence the problem of incomplete drug release and erratic absorption of pure metformin $\mathrm{HCl}$ can be solved by increasing the retention time of drug in GIT for a longer period with the help of mucoadhesive polymers.

\section{Acknowledgement:-}

Authors would like to thank the Department of Pharmacy of University of Asia Pacific for providing facilities during the present research work.

\section{References:-}

1. Boddupalli, B.M., Mohammed, Z.N.K., Nath, R.A. and Banji, D. (2010): Mucoadhesive drug delivery system: an overview. J. Adv. Pharm. Technol. Res., 1(4): 381-387.

2. Smart, J.D. (2005): The basics and underlying mechanisms of mucoadhesion. Adv. Drug Deliver. Rev., 57: $1556-1568$.

3. Hägerstrom, H. and Edsman, K. (2003): Limitations of the rheological mucoadhesion method: the effect of the choice of conditions and the rheological synergism parameter. Eur. J. Pharm. Sci., 18: 349-357.

4. Kadhe, G. and Arasan, R.E. (2002): Advances in drug delivery of oral hypoglycemic agents. Curr. Sci., 83(12): 1539-1543.

5. Desvergne, B. and Wahli, W. (1999): Peroxisome proliferator-activated receptors: nuclear control of metabolism. Endocr. Rev., 20: 649-668.

6. Berger, J. and Moller, D.E. (2002): The mechanisms of action of PPARs. Annu. Rev. Med., 53: 409-435.

7. Chawla, A., Repa, J.J. and Evans, R.M. (2001): Nuclear receptors and lipid physiology: opening the X-files. Science, 294: 1866-1870.

8. Chinetti, G., Fruchart, J.C. and Staels, B. (2000): Peroxisome proliferator-activated receptors (PPARs): nuclear receptors at the crossroads between lipid metabolism and inflammation. Inflamm. Res., 49: 497-505.

9. Willson, T.M., Lambert, M.H. and Kliewer, S.A. (2001): Peroxisome proliferator-activated receptor gamma and metabolic disease. Annu. Rev. Biochem., 70: 341-367.

10. Singh, P.K., Shukla, V.K., Easwari, T.S., Kumar, S., Chaudhary, R. and Saraswat, S. (2012): Formulation development and evaluation of mucoadhesive oral dosage form containing clarithromycin using different mucoadhesive polymers. Int. J. Pharm. Sci.Health Care., 2(2): 166.

11. Umarji, B., Patil, R., Birajdar, R., Mysore, S., Bilagi, S. and Audurti, D. (2012): Formulation and in vitro evaluation of mucoadhesivebuccal tablets of furosemide. WJPPS, 1(3): 1041-1063. 